\title{
Concise Commentary: Quercetin Flavonoid of the Month or IBD Therapy?
}

\section{Toshinori Yoshida $^{1}$}

Published online: 4 September 2018

(c) Springer Science+Business Media, LLC, part of Springer Nature 2018
Quercetin, a naturally occurring polyphenol flavonoid [1], is used as a nutritional supplement for the treatment of cardiovascular diseases, allergy, asthma, obesity, diabetes, cancer, and systemic or local inflammation. Quercetin directly targets cellular substrates regulating apoptosis, the cell cycle, growth arrest, senescence, and inflammation [2]. Antiinflammatory effects of quercetin have been reported in several cell lines including macrophages, glial cells, fibroblasts, and cancer cells in response to proinflammatory compounds such as lipopolysaccharide (LPS) [1,3]. Understanding why quercetin inhibits inflammation is of importance for the area of nutraceutical sciences and related fields.

Inflammatory bowel disease (IBD), one of the most important chronic inflammatory diseases in humans, is associated with functional and morphological changes in the vascular endothelium which are key to IBD pathogenesis [4]. By activating endothelial cells, a variety of cell adhesion molecules (CAMs) such as intercellular CAM-1 (ICAM-1) and vascular CAM-1 (VCAM-1) and chemokines, such as interleukin- $1 \beta$ and -6 , and tumor necrosis factor- $\alpha$ are expressed in or over the cell surface, followed by strongly recruiting leukocytes and promoting inflammatory responses. Human intestinal microvascular endothelial cells (HIMECs) have unique adhesion and growth patterns; in IBD patients, inflamed microvessels display enhanced expression of CAMs and leukocyte adhesion molecules compared with healthy controls [4]. Therefore, experiments using intestinal microvascular endothelial cells from humans and animal models are believed to be an important step for exploring new aspects of the pathogenesis of IBD.

In this issue of Digestive Disease and Sciences, Bian et al. [5] reported that quercetin prevented enhanced ICAM-1 and

Toshinori Yoshida

yoshida7@cc.tuat.ac.jp

1 Laboratory of Veterinary Pathology, Tokyo University of Agriculture and Technology, 3-5-8 Saiwai-cho, Fuchu-shi, Tokyo 183-8509, Japan
VCAM-1 expression in LPS-activated rat intestinal microvascular endothelial cells (RIMVECs). Importantly, the anti-inflammatory effect of quercetin was confirmed by the reduced expression of the LPS receptor Toll-like receptor (TLR)4 and MyD88 expression, in association with altered expression of several inflammatory central regulators such nuclear factor (NF)- $\kappa B$ p $65 / \mathrm{I} \kappa \mathrm{B}-\alpha$, mitogen-activated protein kinases (MAPK)s including extracellular signal-regulated kinase and c-Jun N-terminal kinase but not p38 MAPK, and signal transducer and activator of transcription (STAT). These findings clearly indicated that quercetin can inhibit TLR4-mediated inflammatory responses in LPS-stimulated RIMVECs through the MyD88-dependent NF- $\mathrm{BB}$, MAPKs, and STAT pathways. TLRs in intestinal endothelial cells are activated by LPS derived from Gram-negative bacteria present in the intestinal lumen and surface when mucosal permeability is increased. Therefore, quercetin may prevent the initial activation of the inflammatory cascade by preventing LPS activation of endothelially expressed TLR4. Nevertheless, the authors did not demonstrate the effects of quercetin on RIMVECs when concomitantly or pre-treated with LPS followed by the application of quercetin; therefore, the mechanism underlying the anti-inflammatory effects of quercetin remains incompletely understood.

A well-known drawback of quercetin is its poor oral bioavailability in humans and animals [1]. Flavonoids exist in nature as either glycosides or aglycones with the glycoside form the most commonly present and the aglycone form predominant in plasma. Therefore, the in vitro studies of Bian et al. need to be confirmed in experimental IBD models in order to further understand the anti-inflammatory effects of the flavonoid.

\section{References}

1. D'Andrea G. Quercetin: a flavonol with multifaceted therapeutic applications? Fitoterapia. 2015;106:256-271. 
2. Russo M, Spagnuolo C, Tedesco I, Bilotto S, Russo GL. The flavonoid quercetin in disease prevention and therapy: facts and fancies. Biochem Pharmacol. 2012;83:6-15.

3. Boots AW, Haenen GR, Bast A. Health effects of quercetin: from antioxidant to nutraceutical. Eur J Pharmacol. 2008;585:325-337.

4. Cibor D, Domagala-Rodacka R, Rodacki T, Jurczyszyn A, Mach T, Owczarek D. Endothelial dysfunction in inflammatory bowel diseases: pathogenesis, assessment and implications. World J Gastroenterol. 2016;22:1067-1077.

5. Bian Y, Liu P, Zhong J, Zhuang S, Fan K, Liu Z. Quercetin attenuates adhesion molecules expression in intestinal microvascular endothelial cells by modulating multiple pathways. Dig Dis Sci. (Epub ahead of print). https://doi.org/10.1007/s1062 0-018-5221-2. 